\title{
Gonadal Toxicity and Fertility after Radiotherapy for Testicular Seminoma
}

\author{
J. Claßen M. Bamberg \\ Abteilung für Strahlentherapie, Radiologische Universitätsklinik, Tübingen
}

Due to the exquisite radiosensitivity of seminoma, radiotherapy has been established for decades as routine adjuvant treatment for testicular seminoma. Even with upcoming singleagent carboplatin chemotherapy, irradiation unequivocally remains the standard adjuvant treatment for early stage I and IIA/B disease (Royal Marsden Classification) [1]. With cure rates of $95-98 \%$, early and possible late side effects of radiotherapy gain particular importance. Considerable progress has been made in recent years in reducing the toxicity of adjuvant radiotherapy by refining treatment portals to infradiaphragmal lymph nodes and substantially lowering the total dose of irradiation [2, 3]. Limited para-aortic (PA)/paracaval radiotherapy for stage I seminoma with 26 Gy and dogleg (DL) irradiation, comprising PA/paracaval and ipsilateral iliac lymph nodes with 30 and 36 Gy for stage IIA/B disease, respectively, are currently accepted as standard adjuvant therapy [1]. Nevertheless, there is persistent concern about gonadal toxicity and infertility after radiotherapy since scattered irradiation is well known to severely interact with spermatogenesis in the remaining testis. When discussing post-radiotherapy fertility of seminoma patients, numerous factors like field arrangement, total dose of treatment, and testicular shielding have to be considered as well as the time course of sperm count recovery after treatment and the preexisting post-orchiectomy impairment of spermatogenesis.

\section{Pretreatment Fertility}

After orchiectomy and before adjuvant radiotherapy, approximately $60 \%$ of all patients present with oligo- or azoospermia $[4,5] .20 \%$ of these patients show an additional elevation of serum FSH levels. In their detailed analysis of post-treatment fertility, Jacobsen et al. [5] conclude that the sperm count in those patients presenting with normal pre-treatment spermatograms was likely to recover within $2-3$ years after radiotherapy. This observation is supported by a prospective investigation of Centola et al. [6], who report 8 patients with full pre- and post-treatment sperm count evaluation. All patients were normozoospermic before adjuvant treatment. Subsequent to testicular scattering doses between $0.28-0.65 \mathrm{~Gy}$, all patients regained normal sperm counts within a maximum of 3 years.
Furthermore, Jacobsen et al. [5] suggest that the probability of sperm recovery after treatment was low in those patients whose subnormal pre-treatment spermatograms were paralleled with a highly increased FSH level. However, in patients with no or only moderate FSH elevation and oligo- or azoospermia before treatment, a marked increase in sperm count after radiotherapy could be expected. These findings are supported by Fossa et al. [7] who demonstrated that pre-treatment oligo- or azoospermia do not predict post-treatment infertility $-50 \%$ of patients treated either way showed sperm count recovery after radiotherapy. The authors also conclude that the combination of highly elevated pre-treatment FSH and azoospermia can identify those men with severely impaired spermatogenesis who probably will not recover after adjuvant irradiation. Hence, for individual assessment of fertility and possible medicolegal reasons, a detailed fertility analysis including sperm count and serum FSH is recommended on a routine basis [8].

\section{Testicular Radiosensitivity to Single-Dose Irra- diation}

Early studies with single-dose testicular irradiation showed that doses between 0.08-0.5 Gy resulted in oligozoospermia while azoospermia was observed at doses above 0.5 Gy [9]. Time to recovery after treatment showed a clear dose dependency and at least partial restitution has been reported after single doses above 10 Gy. However, the phases of spermatogenesis show different radiosensitivity and cycle times, with spermatogonia being the most sensitive cell population. This heterogeneity eventually leads to an increased radiosensitivity in the case of fractionated treatment, which in clinical routine therapy is applied rather than single-dose irradiation.

\section{Testicular Radiosensitivity to Fractionated Irra- diation}

Midplane Dose and Gonadal Irradiation Scattering

The prescribed midplane tumor dose of radiotherapy is of obvious importance for the total gonadal dose of radiation

\begin{tabular}{ll}
\hline KARER & @ 1999 S. Karger GmbH, Freiburg \\
& Fax (0761) 4520714 \\
www.karger.com & Accessible online at: \\
http://BioMedNet.com/karger
\end{tabular}

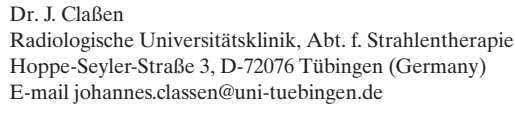


scattering. Hansen et al. [10] performed testicular measurements of dose scattering during fractionated DL irradiation with a median midbeam tumor dose of $40.3 \mathrm{~Gy}$, no testicular shielding was applied. The median dose was 1.7 Gy or $4.3 \%$ of the midline dose. While pretreatment spermatograms revealed oligo- or azoospermia in $51 \%$ of the men, all patients showed subnormal sperm counts after treatment and azoospermia was observed in $84 \%$. Time to recovery was significantly correlated to total dose of gonadal radiation scattering, patient age at the time of treatment, and pretreatment sperm quality. Young men with a high pretreatment sperm count and a scattering dose $\leq 1.3 \mathrm{~Gy}$ had the best chance for quick recovery of fertility within 1-2 years. This observation was supported by Gordon et al. [11], who measured a median testicular dose of 0.79 Gy during abdominal radiotherapy with a midbeam dose of $26 \mathrm{~Gy}$, thus clearly demonstrating the benefit reduced total treatment doses have on testicular scattering. Time to recovery after testicular doses below 0.79 Gy was reduced to approximately 1 year - while doses above 0.79 Gy required 2 years for the recovery of sperm count. Applying a 30 Gy midplane tumor dose, Joos et al. [8] measured a testicular dose between 0.09-0.79 Gy, depending on the alignment of the field. No impairment of spermatogenesis was observed below scattering doses of $0.2 \mathrm{~Gy}$. In conclusion, these studies clearly demonstrate the benefit of reduced total doses of radiotherapy for the sparing of testicular scattering of irradiation.

\section{Field-Edge Distance}

A strong correlation can be expected between the testicular scattering dose and the distance between inferior field edge and the remaining testicle. Phantom and patient measurements clearly support this view $[5,11]$. Measuring the symphysis-to-testicle distance, Jacobsen et al. [5] established a mathematical function predicting the testicular scattering dose for DL and pure PA irradiation. In the same series, patient measurements revealed a total gonadal dose of 0.32 Gy for DL and 0.09 Gy for PA irradiation with a midplane dose of $30 \mathrm{~Gy}$ in 2 Gy daily fractions. Radiation protection was applied only to those patients receiving the DL field; no shielding was used with the PA technique. This study showed a significant reduction in sperm count 1 year after treatment with the DL field, while no impairment of sperm quality was observed after PA irradiation. In patients with a subnormal sperm count before radiotherapy, no further deterioration 1 year after treatment was observed with either. In this series no data were presented for long-term recovery of sperm counts. A similar study was performed by Joos et al. [8], monitoring FSH levels before and after either DL or PA irradiation. A significant rise in FSH levels was demonstrated only in the DL group of patients with a peak 6 months after treatment and a gradual return to normal ranges after 36 months. In the PA group, no rise in FSH levels above the upper normal limit was observed, indicating no significant gonadal dysfunction after low-dose limited abdominal radiotherapy. These observations strongly support the current concept of pure PA irradiation for stage I disease, enabling an optimal sparing of the testicular scattering dose. With low-dose PA radiotherapy, no gonadal toxicity has to be expected and testicular shielding is not required.

\section{Androgen Synthesis}

While the cells of spermatogenesis are highly sensitive to ionizing radiation, Leydig cells are far more radioresistant. Following a report by Izard [12], a decline in androgen synthesis has to be expected only after testicular dose of $33 \mathrm{~Gy}$, an increase in serum luteinizing hormone (LH) can be expected at rates above $14 \mathrm{~Gy}$. Likewise, a series by Fossa et al. [4] failed to demonstrate significant changes in LH or testosterone after a median gonadal dose of 0.548 Gy during DL irradiation. On the other hand, Joos et al. [8] observed a significant rise of serum LH levels within normal limits after 30-Gy DL irradiation, resulting in a mean testicular scattering dose of $0.54 \mathrm{~Gy}$. However, after limited 30-Gy PA irradiation no increase in LH levels was found. These data indicate that a measurable but clinically insignificant and reversible impairment of Leydig cell function may be observed even after low-dose DL abdominal irradiation.

\section{Paternity and Sexual Functioning after Abdominal Irradiation for Seminoma}

In their study of reproductive function, Gordon et al. [11] report on 13 pregnancies fathered by 12 of 53 patients after abdominal irradiation for seminoma. Likewise, Centola et al. [6] observed paternity in 3 of 8 patients after a maximum scattering dose of $0.90 \mathrm{~Gy}$. The children were conceived between 9 and 14 months after treatment. Moreover, they report on another 2 patients who fathered a child 30 and 43 months after radiotherapy with a gonadal scattering dose of up to $1.2 \mathrm{~Gy}$. In a study by Fossa et al. [7], 5 of 6 patients $(83 \%)$ with pretreatment oligozoospermia, who attempted to father a child after adjuvant radiotherapy, actually succeeded. In conclusion, paternity after abdominal radiotherapy for seminoma is feasible as may be expected from the presented data of post-treatment spermatograms and FSH levels.

Only a few studies systematically address the topic of sexual dysfunction after adjuvant radiotherapy for testicular tumor. The overall sexual satisfaction evaluated by Gordon et al. [11] in 32 and 24 men 1 and 2 years after treatment, respectively, was rated as 'good' by the majority of patients. In a more detailed analysis of sexual functioning after treatment for testicular germ cell tumor by Jonker-Pool et al. [13], the patients receiving radiotherapy for seminoma did not significantly differ from those men being followed with a wait-and-see strategy. Approximately $20 \%$ of the patients in both groups reported a decline in $\geq 1$ sexual function with an overall decrease in sexual satisfaction in $7.5 \%$ of the patients in the radiotherapy group. These data clearly indicate that psychological factors play an important role for the self-estimation of sexual functioning after treatment. Sexual satisfaction after radiotherapy can be expected to remain unaffected in the majority of patients.

\section{Conclusions}

Considerable progress has been achieved in modern radiotherapy by reducing treatment volumes and the total dose of irradiation for early-stage seminoma. With low-dose pure PA irradiation for stage I disease, only minimal radiation scatter- 
ing to the remaining testis has to be expected, leaving spermatogenesis unimpaired. Hence, no testicular shielding is required for stage I PA radiotherapy with 26 Gy. After DL irradiation with 30 and 36 Gy, respectively, some transient reduction in sperm quality with stage IIA/B has to be taken into account. For these patients, routine testicular shielding as well as sperm banking is recommended. Patients presenting with a normal pretreatment sperm count can expect complete recovery of fertility within 2 years after radiotherapy. On the other hand, more than $60 \%$ of all men with testicular seminoma show oligo- or azoospermia before adjuvant treatment. Among these patients further treatment-related reduction in sperm count is unlikely. Separate analysis of spermatogram and FSH may provide a means of individually assessing the patient's probability of fertility restitution after radiotherapy. Approximately $50 \%$ of men presenting with low pre-treatment sperm count will show improved spermatograms after treatment. Sperm counts once per year are reasonable for monitoring fertility after radiotherapy. Should no improvement of sperm quality be observed 3 years after treatment, recovery no longer seems to be achievable [14]. Fathering children after low-dose testicular radiation scattering has been documented frequently, and sexual satisfaction will be maintained by the majority of patients. Even though no teratotoxicity after radiotherapy for seminoma has been reported to our knowledge, for safety reasons conception should be avoided for 1 year after treatment.

\section{References}

1 Bamberg M, Schmoll H-J, Weißbach L, Beyer J, Bokemeyer C, Harstrick A, Höltl W, Souchon R, Vogler H: Interdisziplinäre Konsensuskonferenz zur «Diagnostik und Therapie von Hodentumoren». Strahlenther Onkol 1997;173:397-406.

2 Schmidberger H, Bamberg M: Therapieoptionen bei testikulären Seminomen in den frühen Stadien. Strahlenther Onkol 1995;171:125-139.

3 Schmidberger H, Bamberg M, Meisner C, Classen J, Winkler C, Hartmann M, Templin R, Wiegel T, Dornhoff W, Ross D, Thiel HJ, Martini C, Haase W: Radiotherapy in stage IIA and IIB testicular seminoma with reduced portals: A prospective multicenter study. Int J Radiat Oncol Biol Phys 1997;39: 321-326.

4 Fossa SD, Aabyholm T, Normann N, Jetne V: Posttreatment fertility in patients with testicular cancer III. Influence of radiotherapy in seminoma patients. Br J Oncol 1986;58:315-319.
5 Jacobsen KD, Olsen DR, Fossa K, Fossa SD: External beam abdominal radiotherapy in patients with seminoma stage I: Field type, testicular dose, an spermatogenesis. Int J Radiat Oncol Biol Phys 1997;38:95-102.

6 Centola GM, Keller JW, Henzler M, Rubin P: Effect of low-dose testicular irradiation on sperm count and fertility in patients with testicular seminoma J Androl 1994;15:608-613.

7 Fossa SD, Theodorsen L, Normann N, Aabyholm T: Recovery of impaired pretreatment spermatogenesis in testicular cancer. Fertil Steril 1990;54:493-496.

8 Joos H, Sedlmayer F, Gomahr A, Rahim HBK, Frick J, Kogelnik HD, Rettenbacher L: Endocrine profiles after radiotherapy in stage I seminoma: Impact of two different radiation treatment modalities. Radiother Oncol 1997;43:159-162.

9 Rowley MJ, Leach DR, Warner GA, Heller CG Effect of graded doses of ionizing radiation on the human testis. Radiat Res 1974:59:665-678.

10 Hansen PV, Trykker H, Svennekjaer IL, Hvolby J: Long-term recovery of spermatogenesis after radiotherapy in patients with testicular cancer. Radiother Oncol 1990;18:117-125.
11 Gordon W, Siegmund K, Stanisic TH, McKnight B, Harris IT, Carroll PR, Paradelo JC, Meyers FR, Chapman RA, Meyskens FL: A study of reproductive function in patients with seminoma treated with radiotherapy and orchiectomy: (SWOG-8711). Int J Radiat Oncol Biol Phys 1997;38:83-94.

12 Izard MA: Leydig cell function and radiation: A review of the literature. Radiother Oncol 1995;34:1-8.

13 Jonker-Pool G, van Basten JP, Hoekstra HJ, van Driel MF, Sleijfer DT, Koops HS, van de Wiel HB: Sexual functioning after treatment for testicular cancer: Comparison of treatment modalities. Cancer 1997;80:454-464.

14 Herrmann T: Strahlenreaktionen an den Gonaden Konsequenzen für die Patientenberatung. Strahlenther Onkol 1997;173:493-501. 